\title{
Central Hyperthyroidism
}

National Cancer Institute

\section{Source}

National Cancer Institute. Central Hyperthyroidism. NCI Thesaurus. Code C113146.

Overproduction of thyroid hormones due to a disorder originating within the hypothalamic-pituitary axis. 